\title{
Sympatric speciation in Nicaraguan crater lake cichlid fish
}

\author{
Marta Barluenga ${ }^{1 \star}$, Kai N. Stölting ${ }^{1 \star}$, Walter Salzburger ${ }^{1,2 *}$, Moritz Muschick $^{1} \&$ Axel Meyer $^{1}$
}

Sympatric speciation, the formation of species in the absence of geographical barriers, remains one of the most contentious concepts in evolutionary biology. Although speciation under sympatric conditions seems theoretically possible ${ }^{1-5}$, empirical studies are scarce and only a few credible examples of sympatric speciation exist ${ }^{6}$. Here we present a convincing case of sympatric speciation in the Midas cichlid species complex (Amphilophus sp.) in a young and small volcanic crater lake in Nicaragua. Our study includes phylogeographic, population-genetic (based on mitochondrial DNA, microsatellites and amplified fragment length polymorphisms), morphometric and ecological analyses. We find, first, that crater Lake Apoyo was seeded only once by the ancestral high-bodied benthic species Amphilophus citrinellus, the most common cichlid species in the area; second, that a new elongated limnetic species (Amphilophus zaliosus) evolved in Lake Apoyo from the ancestral species (A. citrinellus) within less than $\sim 10,000 \mathrm{yr}$; third, that the two species in Lake Apoyo are reproductively isolated; and fourth, that the two species are eco-morphologically distinct.

Ever since Darwin proposed the concept in The Origin ${ }^{7}$, the issue of whether sympatric speciation is common or even possible in nature has received much attention from evolutionary biologists. Despite current enthusiasm in favour of sympatric speciation and models espousing that it is theoretically possible ${ }^{1-5}$, the empirical evidence for it remains scant and many of the proposed cases are equally compatible with allopatric schemes. A thorough examination $^{6}$ has led to the conclusion that even the most promising examples of sympatric speciation remain questionable because of the exclusive use of mitochondrial DNA (mtDNA) ${ }^{8,9}$, small sample sizes $^{8,9}$ or insufficient outgroup sampling ${ }^{10}$. It has been emphasized ${ }^{6}$ that a firm corroboration for the origin of one or more species in sympatry would need to fulfil the following criteria: first, a sympatric distribution of the most closely related sister species; second, genetic evidence for reproductive isolation among them; third, their monophyly; and fourth, an ecological setting in which allopatric differentiation is unlikely, such as remote oceanic islands, hosts for parasites or small lakes.

Crater Lake Apoyo in Nicaragua (Fig. 1) provides an exceptionally clear situation for testing sympatric speciation. It is small (diameter, $\sim 5 \mathrm{~km}$; max. depth, $\sim 200 \mathrm{~m}$ ), is of recent origin ${ }^{11}(<23,000 \mathrm{yr})$, has a homogenous habitat (Supplementary Fig. 1), and is completely isolated. This filled caldera has an impoverished fauna compared with nearby water bodies ${ }^{12}$, but contains the widespread Midas cichlid (A. citrinellus, Günther 1864) and an endemic species, the Arrow cichlid (A. zaliosus, Barlow 1976). These, together with Amphilophus labiatus from the large Nicaraguan lakes ${ }^{13-15}$, and possibly more species from some of the crater lakes ${ }^{16}$, make up the Midas cichlid species complex that is restricted to Nicaragua and the north of Costa Rica. In A. citrinellus of other lakes and in A. labiatus, two colour morphs are recognized, but such polymorphism has not been described in Lake Apoyo. To examine whether the endemic Arrow cichlid emerged in Lake Apoyo from an ancestral stock of Midas cichlids under fully sympatric circumstances, we adopted a comprehensive approach including phylogeographic, populationgenetic, morphometric and ecological analyses. We included about 120 specimens from Lake Apoyo and over 500 individuals from six other lakes of Nicaragua (outgroup sample; see Supplementary Table 1).

Analysis of the mitochondrial control region ( $840 \mathrm{bp}$ ) showed that the two Amphilophus species from Lake Apoyo form a monophyletic assemblage (Fig. 2a). Notably, not a single mtDNA haplotype is
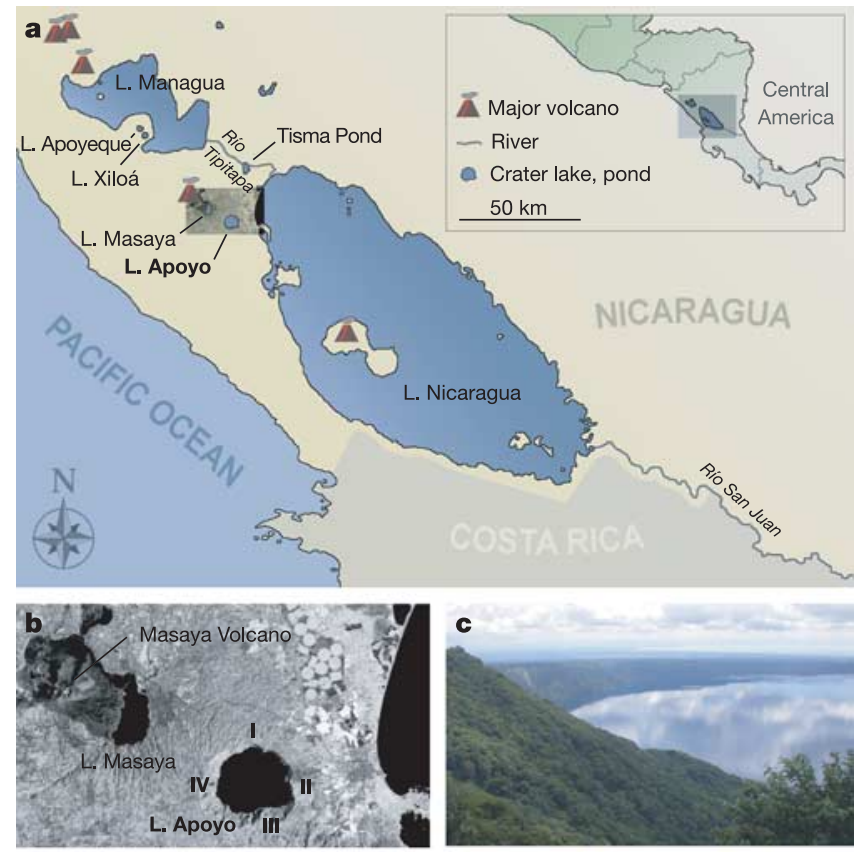

Figure 1 | The study area. a, Map of the Pacific coast of Nicaragua and Costa Rica showing the large Nicaraguan lakes (Managua, Nicaragua), some of the volcanic crater lakes (Apoyo, Apoyeque, Masaya and Xiloá), Tisma Pond and the rivers Tipitapa and San Juan that are home to the Midas cichlid species complex (Amphilophus sp.). b, Satellite image showing Lake Apoyo and neighbouring Lake Masaya that has partially been overflown by lava from a more recent eruption of the Masaya Volcano. Roman numerals indicate the four sampling localities. c, Photograph of Lake Apoyo showing its steep crater wall. 

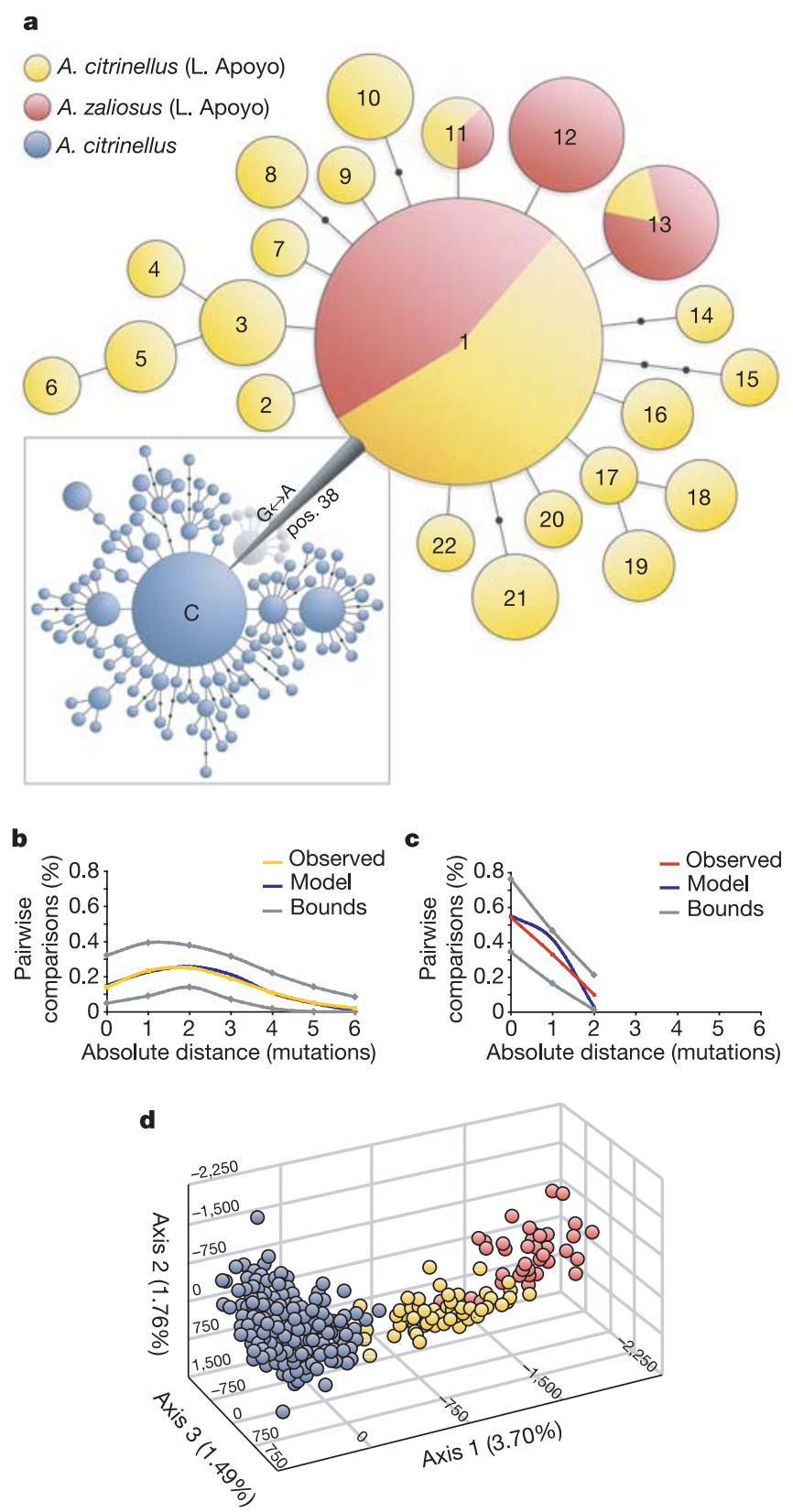

Figure 2 Phylogeography of the Lake Apoyo Amphilophus species. a, Unrooted haplotype network based on $637 \mathrm{mtDNA}$ sequences. Haplotypes are coloured according to species and geographic origin: A. zaliosus (red), A. citrinellus from Lake Apoyo (yellow), and A. citrinellus from other lakes (blue). The framed haplotype network is taken from ref. 15 and is based on 519 specimens from all lakes except Lake Apoyeque (the 30 Lake Apoyo specimens included in that study are shown in grey). The new Lake Apoyo haplotypes are enlarged; their sizes reflect the number of specimens sharing the same haplotype (for example: haplotype 1, 65 individuals; haplotype 2, 1 individual). Lake Apoyo representatives, albeit belonging to two species, form a monophyletic assemblage showing a characteristic mutation in alignment position 38 . The central haplotype in Lake Apoyo (1) is connected by this characteristic mutation to the central haplotype C. b, Coalescence-based mitochondrial mismatch analysis of Lake Apoyo A. citrinellus uncovers a demographic expansion about two mutations ago. c, Mismatch analysis of $A$. zaliosus suggests ongoing demographic expansion (see Supplementary Table 2 for parameters). d, Three-dimensional representation of a factorial correspondence analysis based on microsatellite genotypes. Individuals are colour-coded as described above. shared between Lake Apoyo and any of the remaining lakes, suggesting that there was a single colonization event by an ancestral lineage of $A$. citrinellus and ruling out any contemporary genetic exchange or secondary colonizations. The genetic diversity and the number of mtDNA haplotypes are low in Lake Apoyo (22 versus 125 in the outgroup sample), in line with the young age of the species assemblage $^{15}$. A mitochondrial mismatch analysis indicates a single demographic expansion of $A$. citrinellus in Lake Apoyo about two mutations ago (Fig. 2b), whereas a more recent demographic expansion is detected for the new species A. zaliosus (Fig. 2c and Supplementary Table 2). A plot of the microsatellite distances (Fig. 2d and Supplementary Fig. 2 and Table 3) and the phylogenies based on microsatellites and amplified fragment length polymorphisms (AFLPs; Supplementary Fig. 3) corroborate the distinctive genetic composition of Lake Apoyo's Amphilophus fauna, its monophyly and the evolution of A. zaliosus from the A. citrinellus stock of Lake Apoyo.

Significant $F$-statistics based on all genetic markers show that A. citrinellus and A. zaliosus are reproductively isolated in Lake Apoyo (Table 1). Similarly, microsatellite-based bayesian population assignment tests (Fig. 3) unambiguously identified three clusters: the outgroup sample, A. citrinellus and A. zaliosus from Lake Apoyo. Reproductive isolation is further suggested by mate-choice experiments that demonstrate strong assortative mating between both species $^{17}$.

The two Amphilophus species in Lake Apoyo are morphologically clearly distinct ${ }^{13,14}$ (Fig. 4a). Morphometric analysis uncovered two discrete body types corresponding to the two species, with body height explaining most of the differences (Fig. 4b). The two species also differ in the shape of a trophically relevant structure that is tightly linked to the ability of cichlids to process alternative food types ${ }^{18}$ : the pharyngeal jaw (Fig. 4c). Morphometric analyses show clear differences between the two species (Fig. 4d), with A. zaliosus having more elongated pharyngeal jaws. Stomach content analyses indicate significant dietary differences between the two species and a wider trophic niche in A. citrinellus (Fig. 4e). Our eco-morphological inferences characterize $A$. citrinellus as benthic forager with a deeper body, and A. zaliosus as limnetic form with an elongated trunk that appears to be adapted to living in the open water column.

Taken together, we present a strong case where only sympatric speciation can account for the origin of a new species from a more widespread one in Lake Apoyo in $<10,000 \mathrm{yr}$ (Supplementary Table 2). The Lake Apoyo population of the Midas cichlid and the Arrow cichlid form a monophyletic assemblage (Fig. 2 and Supplementary Fig. 3); they are reproductively completely isolated as shown by mate-choice experiments ${ }^{17}$ and the analyses of three sets of molecular markers (Table 1); they are sympatrically distributed, no genetic structuring was detected in A. citrinellus and A. zaliosus, and even individuals from opposite sides of the crater lake show no sign of differentiation (Supplementary Table 4, Supplementary Fig. 4). The recent volcanic origin of Lake Apoyo, its small size, its degree of isolation, the homogeneous habitat and the sympatric occurrence of both species throughout the lake, as well as the absence of genetic structure in each of the two species rule out the possibility of (micro-) allopatric or parapatric differentiation.

Disruptive natural selection is likely to explain the evolution of alternative habitat preferences in Lake Apoyo's cichlid species.

Table 1 | Genetic distance between the two Amphilophus species from Lake Apoyo

\begin{tabular}{lccc}
\hline F-statistics & $\begin{array}{c}\text { mtDNA } \\
(840 \mathrm{bp})\end{array}$ & $\begin{array}{c}\text { Microsatellites } \\
(10 \text { loci })\end{array}$ & $\begin{array}{c}\text { AFLPs } \\
\text { (226 variable loci) }\end{array}$ \\
\hline A. citrinellus versus A. zaliosus & $0.06^{\star \star}$ & $0.12^{\star \star}$ & $0.06^{\star \star}$ \\
\hline
\end{tabular}

The genetic distance was measured with $F$-statistics $\left.{ }^{\star \star} P<0.001\right)$. 


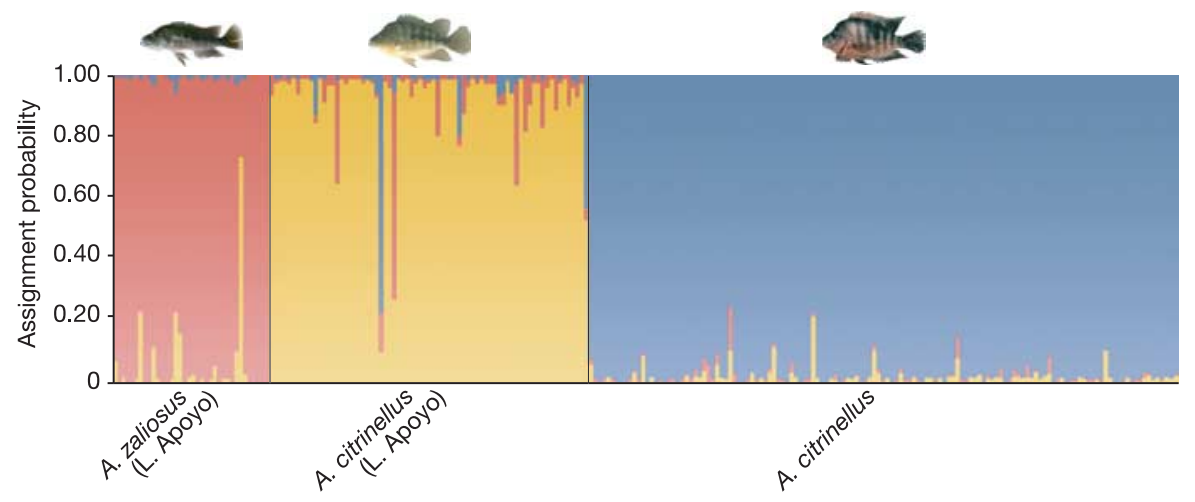

Figure 3 | Bayesian population assignment test. A population assignment test with the software Structure based on ten microsatellite loci uncovered

three distinct populations (A. zaliosus, red; A. citrinellus from Lake Apoyo, yellow; and A. citrinellus from Lake Nicaragua, blue).

However, most theoretical models predict that the completion of speciation requires the evolution of other forms of pre- or postzygotic isolation ${ }^{5}$, such as assortative mating, and a linkage disequilibrium between loci involved in disruptive natural selection and those involved in mate choice ${ }^{1,3}$. Both field observations ${ }^{13}$ and laboratory experiments ${ }^{17}$ show that the two Amphilophus species in Lake Apoyo mate assortatively. Although heterospecific pairs can form, they do not lead to successful matings ${ }^{17}$, indicating that differences in courtship behaviour result in effective prezygotic isolation. Therefore, both ecological speciation mechanisms through divergent habitat preferences and resource partitioning, and assortative mating through behavioural isolation separate the two Amphilophus species in Lake Apoyo.

The finding that the two Amphilophus species from Lake Apoyo differ in eco-morphological traits and resource utilization (Fig. 4) indicates that ecological disruptive selection played a principal part in the speciation event. Similar processes of divergent niche occupation are also known from other species of freshwater fishes ${ }^{19}$. For Lake Apoyo, it seems likely that a new ecological dimension-the open water column over greater depths, which is absent in the shallow large Nicaraguan lakes-facilitated the emergence of a limnetic morphotype and, ultimately, of the new species A. zaliosus. In this respect it is interesting that in the second deepest lake in Nicaragua, Crater Lake Xiloá (maximum estimated depth, $100 \mathrm{~m}$ ), an elongated morphotype of the Midas cichlid occurs that also seems to be adapted to the open water column, but is not related to A. zaliosus ${ }^{15,16}$. Although details about ecology, morphology and genetic structure of Lake Xiloás elongated morphotype remain tentative, it seems plausible that the Xiloa situation parallels the one in Lake Apoyo, rendering the Midas cichlid assemblage as another example of parallel sympatric speciation in addition to the sticklebacks in postglacial lakes in British Columbia ${ }^{19}$.

The outstanding species richness of cichlids in the East African Great Lakes, which alone contain more than 1,500 endemic species $^{20,21}$, has repeatedly been suggested to be caused by sympatric speciation through sexual selection, as most cichlid species differ more strongly in body coloration than in morphology ${ }^{22,23}$. However, the enormous dimensions of these lakes, their habitat complexity, and the complicacy to infer sister species relationships among closely related taxa in those systems make it difficult, if not impossible, to discard allopatric or parapatric schemes. But one of the few possible cases of sympatric speciation involves cichlids, namely tilapiine cichlids in crater lakes in Cameroon ${ }^{6,8,24}$, where, as in Lake Apoyo, disruptive natural selection seems to be the driving force of the formation of new species. This is in line with data showing that sympatric speciation by sexual selection alone is rather unlikely ${ }^{25,26}$. The finding that sympatric speciation accounts for the origin of at least one new species in the Midas cichlids, and probably also for the emergence of new species in West African crater lakes, again under- scores the special propensity of cichlids for sympatric speciation. Further empirical studies, new theoretical approaches and, in particular, the synthesis of empirical data and simulations are necessary to justify the extrapolation from a small model system such as Lake Apoyo to the exceptionally diverse cichlid species flocks of East African lakes Victoria, Malawi and Tanganyika.

\section{METHODS}

Sampling. A detailed list of all specimens included in this study, voucher identifications, sampling localities, mtDNA haplotypes and GenBank accession numbers is provided in Supplementary Table 1. Fish were collected in the fall of 2003 with gill nets and photographed. Fin clips preserved in $90 \%$ ethanol were taken as DNA samples. Both Lake Apoyo species co-occurred at all sampling sites (Fig. 1 and Supplementary Table 1). Fish heads were taken as voucher specimens and to dissect the lower pharyngeal jaws. In addition, full stomachs of some sampled individuals were taken for dietary analyses. Sampling was done with permit 033-0903 of the Nicaraguan Ministry of Natural Resources (MARENA)

Stomach content analysis. The stomachs were dissected using a dissecting stereomicroscope, and the contents were classified into five categories: biofilm (amorphous blue-green substance mixed with sand ${ }^{12}$ ), the algae Chara and other plant material, zooplankton, insects and fish remnants. The volume of each category present in each individual was estimated and used to calculate Schoener's index of diet overlap ${ }^{27}$ (values greater than 0.6 generally indicate biologically significant overlap) and trophic niche breadth ${ }^{28}$ of both species.

Morphometric analyses. Nine landmarks describing the body shape of the fish from Lake Apoyo (81 A. citrinellus, 35 A. zaliosus) and ten landmarks describing the shape of their pharyngeal jaws ( $30 \mathrm{~A}$. citrinellus, $18 \mathrm{~A}$. zaliosus) were digitized from images taken with a Nikon Coolpix 995 digital camera using TpsDig2 (ref. 29). Two-dimensional Procrustes fitting was done with Past ${ }^{30}$ to standardize landmark coordinates, followed by a shape principal component analysis (PCA) to identify the variables that accounted for the largest variance in the data. Species were discriminated on the basis of established meristic measurements ${ }^{13}$.

mtDNA analyses. Total DNA was extracted by standard salt extraction and the whole control region was amplified by using published primers and PCR conditions ${ }^{15}$. A haplotype network was constructed from the maximum-likelihood tree constructed with Paup* $4.0 \mathrm{~b} 10$ (http://www.sinauer.com) that was translated into maximum parsimony branch lengths. A coalescence-based mismatch analysis was done with Arlequin 2.1 (http://gb.unige.ch/arlequin) to study the demographic history and to estimate the relative timing of the population expansion of the fish in Lake Apoyo.

Microsatellite genotyping. Individuals were genotyped with ten unlinked microsatellite loci (those in ref. 15, and Unh011, Unh012 and Unh013; ref. 16), amplified with fluorescently labelled reverse primers (HEX and FAM dyes), and analysed on an ABI 3100 automatic capillary sequencer. Fragment visualization and scoring were done as previously described ${ }^{15}$.

AFLP amplification. AFLP analysis was done by standard protocols using a plant mapping kit (Applied Biosystems). Five primer combinations were used for selective amplification (EcoRI + /MseI + : ACA/CAA, ACA/CTG, ACC/CAA, ACC/CAG, AGC/CAA); the EcoRI primers were fluorescently labelled (FAM). Visualization and scoring of fragments were done as described for microsatellites. The presence or absence of fragments was binary coded ( 1 or 0 ), 
a
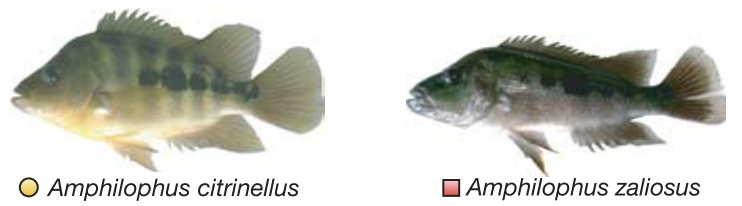

b
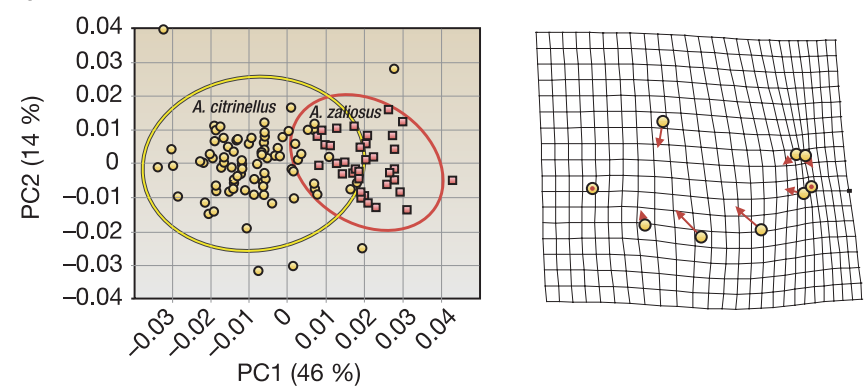

c
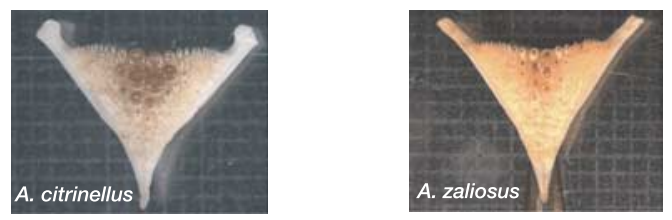

d
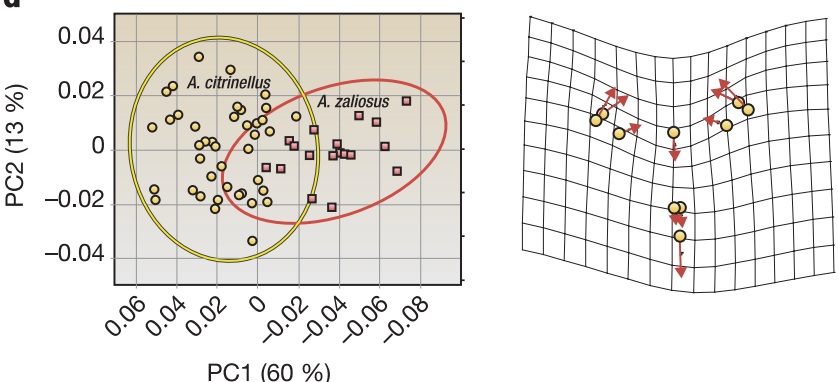

e
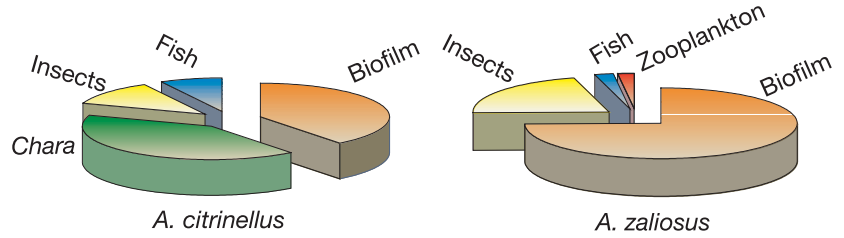

Figure 4 | Eco-morphological assessment of Lake Apoyo's

Amphilophus sp. a, The Midas cichlid (Amphilophus citrinellus) and the Arrow cichlid (A. zaliosus) are morphologically distinct. b, A PCA based on nine landmarks (left) shows a clear separation in body shape between A. citrinellus and A. zaliosus with little overlap. Ellipses represent the $95 \%$ confidence intervals. The transformation grid based on principal component 1 (right; scaling factor 2) indicates that body height accounts for most of the variance between the two species. c, $A$. citrinellus and A. zaliosus show clear differences in their pharyngeal jaws. d, A PCA based on ten landmarks (left) shows clear differences in this structure between the two species. The corresponding transformation grid (right; scaling factor 2) indicates that the pharyngeal jaws of $A$. zaliosus are more elongated. In addition, $A$. zaliosus has only relatively small pointed teeth, whereas some A. citrinellus have molariform teeth. e, Stomach content analyses reveal clear-cut differences between the two Lake Apoyo species (Schoener index: 0.53). Both species forage on biofilm, but whereas winged insects were found in all individuals of $A$. zaliosus, insects were recovered in only about half of the A. citrinellus stomachs. Instead, the latter feed on the algae Chara and other plant material that is found close to the lake's shore ${ }^{12}$. The feeding niche of $A$. citrinellus is wider than that of A. zaliosus (Levins index: $B_{\text {citrinellus }}=3.01, B_{\text {zaliosus }}=1.85$ ). and a distance matrix was constructed with Arlequin for statistical analyses. Population genetic parameters and phylogenetic reconstruction. Allelic diversity, genetic variation, deviation from Hardy-Weinberg equilibrium, and genetic differentiation were calculated with Arlequin. F-statistics were calculated for all molecular markers as implemented in Arlequin. Significance levels were Bonferroni-corrected. Using microsatellite data, we applied a bayesian model-based clustering algorithm implemented in Structure 2.1 (http:// pritch.bsd.uchicago.edu) to test the assignment of the cichlids from Lake Apoyo. We used the admixture model and determined the number of ancestral clusters, $K$, by comparing log-likelihood ratios in multiple runs for values of $K$ between 1 and 5. Each run consisted of 1,000,000 iterations with a burn-in period of 50,000; multiple runs with the same value of $K$ led to virtually identical results. To detect the degree of similarity of the Midas cichlids in Lake Apoyo to those outside the lake, a factorial correspondence analysis of the microsatellite data was done with Genetix 4.0 (http://www.univ-montp2.fr/ genetix/genetix/ genetix.htm), which graphically projects the individuals on the factor space defined by the similarity of their allelic states. We inferred a phylogeny of different populations of Midas cichlids on the basis of the microsatellite data with Contml of the Phylip package (http://evolution.genetics.washington.edu/ phylip.html), applying a brownian motion approximation using the mean allelic copy number, and with Populations (http://www.cnrs-gif.fr/pge/ bioinfo/populations/index.php) using the Cavalli-Sforza and Edwards method (10,000 bootstrap replicates on locus information). For both analyses individuals were grouped according to species and lake of origin. We used Paup ${ }^{*}$ to reconstruct a phylogeny based on AFLP data (neighbour-joining algorithm; Nei-Li restriction site differences).

To test for the absence of genetic structuring in Lake Apoyo species, we calculated pairwise $F$-statistics among fish from the four sampling localities with Arlequin (we omitted the data of site I of A. zaliosus owing to the small sample size). In addition, we did a bayesian analysis of population structure with Structure for each species. We also ran Migrate (http://popgen. csit.fsu.edu/) to estimate the number of migrants between sample sites within each species.

\section{Received 13 September; accepted 10 October 2005}

1. Dieckmann, U. \& Doebeli, M. On the origin of species by sympatric speciation. Nature 400, 354-357 (1999).

2. Higashi, M., Takimoto, G. \& Yamamura, N. Sympatric speciation by sexual selection. Nature 402, 523-526 (1999).

3. Kondrashov, A. S. \& Kondrashov, F. A. Interactions among quantitative traits in the course of sympatric speciation. Nature 400, 351-354 (1999).

4. Gavrilets, S. Fitness Landscapes and the Origin of Species: Monographs in Population Biology vol. 41 (Princeton Univ. Press, Princeton, NJ, 2004).

5. Kawecki, T. J. in Adaptive Speciation (Dieckmann, Doebeli, Metz \& Tautz, Cambridge Univ. Press, Cambridge, UK, 2004).

6. Coyne, J. A. \& Orr, H. A. Speciation (Sinauer Associates, Sunderland, MA, 2004).

7. Darwin, C. On the Origin of Species by Means of Natural Selection or the Preservation of Favoured Races in the Struggle for Life (J. Murray, London, 1859).

8. Schliewen, U., Tautz, D. \& Pääbo, S. Sympatric speciation suggested by monophyly of crater lake cichlids. Nature 368, 629-632 (1994).

9. Weiblen, G. D. \& Bush, G. L. Speciation in fig pollinators and parasites. Mol. Ecol. 11, 1573-1578 (2002)

10. Gíslason, D., Ferguson, M. M., Skúlason, S. \& Snorasson, S. S. Rapid and coupled phenotypic differentiation in Icelandic Arctic charr (Salvelinus alpinus). Can. J. Fish Aquat. Sci. 56, 2229-2234 (1999).

11. Bice, D. Quaternary volcanic stratigraphy of Managua, Nicaragua: Correlation and source assignment for multiple overlapping plinian deposits. Geol. Soc. Am. Bull. 96, 553-566 (1985).

12. Barlow, G. W. in Investigations of the Ichthyofauna of Nicaraguan Lakes (ed. Thorson, T. B.) 333-358 (Univ. Nebraska Press, Nebraska, 1976).

13. Barlow, G. W. \& Munsey, J. W. in Investigations of the Ichthyofauna of Nicaraguan Lakes (ed. Thorson, T. B.) 359-369 (Univ. Nebraska Press, Nebraska, 1976).

14. Klingenberg, C. P., Barluenga, M. \& Meyer, A. Body shape variation in cichlid fishes of the Amphilophus citrinellus species complex. Biol. J. Linn. Soc. 80, 397-408 (2003).

15. Barluenga, M. \& Meyer, A. The Midas Cichlid species complex: incipient sympatric speciation in Nicaraguan cichlid fishes? Mol. Ecol. 13, 2061-2076 (2004).

16. McKaye, K. R. et al. Behavioral, morphological and genetic evidence of divergence of the Midas cichlid species complex in two Nicaraguan crater lakes. Cuadernos de Investigación de la UCA 12, 19-47 (2002).

17. Baylis, J. R. A quantitative study of long-term courtship: I. Ethological isolation between sympatric populations of the midas cichlid, Cichlasoma citrinellum, and the arrow cichlid, C. zaliosum. Behaviour 59, 59-69 (1976). 
18. Meyer, A. Costs and benefits of morphological specialization: feeding performance in the trophically polymorphic Neotropical cichlid fish, Cichlasoma citrinellum. Oecologia 80, 431-436 (1989).

19. Schluter, D. \& McPhail, J. D. Character displacement and replicate adaptive radiation. Trends Ecol. Evol. 8, 197-200 (1993)

20. Fryer, G. \& Iles, T. D. The Cichlid Fishes of the Great Lakes of Africa. Their Biology and Evolution (Oliver \& Boyd, Edinburgh, 1972).

21. Kocher, T. D. Adaptive evolution and explosive speciation: the cichlid model. Nature Rev. Genet. 5, 288-298 (2004).

22. van Oppen, M. J. H. et al. Assortative mating among rock-dwelling cichlid fishes supports high estimates of species richness for Lake Malawi. Mol. Ecol. 7, 991-1001 (1998).

23. Seehausen, O. \& van Alphen, J. J. M. Can sympatric speciation by disruptive sexual selection explain rapid evolution of cichlid diversity in Lake Victoria? Ecol. Lett. 2, 262-271 (1999)

24. Schliewen, U. et al. Genetic and ecological divergence of a monophyletic cichlid species pair under fully sympatric conditions in Lake Ejagham, Cameroon. Mol. Ecol. 10, 1471-1488 (2001)

25. Arnegard, M. E. \& Kondrashov, A. S. Sympatric speciation by sexual selection alone is unlikely. Evolution 58, 222-237 (2004).

26. Kirkpatrick, M. \& Nuismer, S. L. Sexual selection can constrain sympatric speciation. Proc. R. Soc. Lond. B. 271, 687-693 (2004).

27. Schoener, T. W. Non-synchronous spatial overlap of lizards in patchy habitats. Ecology 60, 703-710 (1970).
28. Levins, R. Evolution in Changing Environments. Some Theoretical Explorations (Princeton Univ. Press, Princeton, NJ, 1968)

29. Rohlf, F. J. TpsDig. (Dept. Ecology and Evolution, SUNY, New York, 2004).

30. Hammer, Ø., Harper, D. A. T.\& Ryan, P. D. Past: paleontological statistics software package for education and data analysis. Palaeontologia Electronica 4 art. 4 (2004).

Supplementary Information is linked to the online version of the paper at www.nature.com/nature.

Acknowledgements We thank the Ministry of Natural Resources (MARENA) in Nicaragua for collection permits; Z. Pérez for support in the field;

C. Chang-Rudolf, K. Hofmann, Y. Chiari and N. Feiner for technical assistance in the laboratory; and S. Gavrilets, T. Price and E. B. Taylor for comments on the manuscript. This work was funded by the Deutsche Forschungsgemeinschaft priority program 1127 ('Adaptive radiations').

Author Information GenBank accession numbers for all mitochondrial contro region sequences can be found in Supplementary Table 1. Reprints and permissions information is available at npg.nature.com/reprintsandpermissions The authors declare no competing financial interests. Correspondence and requests for materials should be addressed to A.M.

(Axel.Meyer@uni-konstanz.de). 